\title{
O cuidado de crianças com necessidades especiais em foco: o olhar de enfermeiros em unidades de terapia intensiva
}

\author{
The care of children with special needs in focus: the view of nurses \\ in intensive care units
}

\author{
Mikaele Aparecida de Paula Araújo' • Cibelly Aliny Siqueira Lima Freitas² • Maria Adelane Alves Monteiro da \\ $\mathrm{Silva}^{3} \bullet$ Etelvina Sampaio Melo ${ }^{4}$ Géssica Fernanda Martins da Silva ${ }^{5}$
}

\begin{abstract}
RESUMO
Os avanços da tecnologia em Unidades de Terapia Intensiva Neonatal aumentaram a sobrevida de crianças com necessidades especiais de saúde (CRIANES), que se constituem um importante público para a assistência de enfermagem. Assim, o objetivo deste estudo foi analisar a percepção de enfermeiros frente ao cuidado de CRIANES e suas famílias em Unidades de Terapia Intensiva Neonatal e Pediátrica. Trata-se de um estudo descritivo-exploratório de abordagem qualitativa, desenvolvido com 10 enfermeiros que atuam nas Unidade de Terapia Intensiva Neonatal e Unidade de Terapia Intensiva Pediátrica em um hospital de referência da zona norte do Ceará, por meio da aplicação de uma entrevista semiestruturada nos meses de maio e junho de 2019. Os resultados evidenciaram a necessidade de sensibilizar o profissional desde a sua formação acadêmica para o cuidado às crianças com necessidades especiais e suas famílias. Ficou evidente a necessidade de ampliar as vivências práticas durante a formação do enfermeiro, uma vez que este exerce papel relevante no desenvolvimento de competências do cuidador familiar para atender as demandas das crianças no processo de reabilitação. Contudo, a qualificação de profissionais numa perspectiva humana e integral é imprescindível para a inclusão das CRIANES e suas famílias na sociedade.
\end{abstract}

Palavras-chave: CRIANES. Família. Deficiência. Enfermagem

\begin{abstract}
Advances in technology in Neonatal Intensive Care Units have increased the survival of children with special health needs (CRIANES), who constitute an important public for nursing care. Thus, the aim of this study was to analyze the perception of nurses regarding the care of CRIANES and their families in Neonatal and Pediatric Intensive Care Units. It is a descriptive-exploratory study with a qualitative approach, developed with 10 nurses who work in the Neonatal Intensive Care Unit and Pediatric Intensive Care Unit in a reference hospital in the north of Ceará, through the application of a semi-structured interview. in the months of May and June 2019. The results showed the need to sensitize the professional since his academic training to care for children with special needs and their families. It became evident the need to expand practical experiences during the training of nurses, since they play an important role in the development of skills of family caregivers to meet the demands of children in the rehabilitation process. However, the qualification of professionals in a human and integral perspective is essential for the inclusion of CRIANES and their families in society. Keywords: Children with special health needs (CRIANES). Family. Deficiency. Nursing
\end{abstract}

I Enfermeira. Residente em Neonatologia na Santa Casa de Misericórdia de Sobral. Email: mikaeleenf7@gmail.com. Lattes: http://lattes.cnpq.br/996222307042I290

2 Enfermeira. Pós-doutorado em Enfermagem pela Universidade Federal do Paraná (UFPR). Docente do Curso de Enfermagem da Universidade Estadual Vale do Acaraú (UVA). Email: cibellyaliny@gmail.com. Lattes: http://lattes.cnpq.br/92709I3804724785

3 Enfermeira. Doutora em enfermagem pela Universidade Federal do Ceará (UFC). Docente do curso de Enfermagem da Universidade Estadual Vale do Acaraú (UVA). Email:Adelanemonteiro@hotmail.com. Lattes: http://lattes.cnpq.br/7385/43792598274

4 Enfermeira. Mestre em Saúde da Família pela Universidade Federal do Ceará (UFC). Enfermeira assistencialista do Centro de Apoio e Diagnóstico do Hospital Regional Norte (HRN). Email: etelvinamelo@hotmail.com. Lattes: http://lattes.cnpq.br/5910065368503023

5 Graduanda em Enfermagem pela Universidade EstadualVale doAcaraú (UVA).Email:gessica_martins I@hotmail.com.Lattes:http://lattes.cnpq.br/6I28I78I5I568322 


\section{INTRODUÇÃO}

Segundo os dados do último censo do IBGE (Instituto Brasileiro de Geografia e Estatística) em 2010, 45.606.048 brasileiros, $23,9 \%$ da população total, têm algum tipo de deficiência visual, auditiva, motora e mental ou intelectual. As deficiências podem ser originadas no período gestacional, como consequência do parto, ou no período neonatal. Doenças transmissíveis como Zica, Rubéola, Citomegalovirose, Parvovirose, Varicela, HIV, Sífilis, Herpes, Toxoplasmose, Hepatite, entre outras, são causas de origem de algumas deficiências, além de doenças crônicas, perturbações psiquiátricas, desnutrição, abusos de drogas, traumas e lesões'.

Os avanços da tecnologia em Unidades de Terapia Intensiva Neonatal aumentaram a sobrevida de crianças com condições de saúde graves. As crianças com necessidades especiais de saúde (CRIANES), assim denominadas no Brasil e na literatura internacional pelo Maternal and Child Health Bureau (MCHB) como Children with Special Health Care Needs (CSHCN), demandam um acompanhamento em longo prazo em toda a rede de saúde. As CRIANES são aquelas que possuem ou estão em maior risco de apresentar uma condição crônica, tanto física, quanto de desenvolvimento, comportamento ou emocional, o que requer uma especificidade diferenciada atendimento, pelos serviços de saúde, para além daquela geralmente requerida por outras crianças ${ }^{2}$.

Numa pesquisa realizada com profissionais da área da saúde, em relação as experiências vivenciadas no momento de comunicar o diagnóstico da deficiência do recém-nascido para os pais, os autores destacam que a forma com que a informação é dada a família, irá contribuir de forma positiva, ou não, para o processo de adaptação da família em relação aos cuidados à criança com deficiência. Diversos problemas, anseios e dúvidas que deveriam ser resolvidos por meio de uma orientação adequada da equipe de saúde à família da criança com deficiência, acabam acarretando dificuldades para o cuidado à criança no domicílio, após a alta hospitalar. Desta forma, a conscientização precoce e adequada dos pais sobre o diagnóstico do filho, deve ser priorizada pelos profissionais de saúde ${ }^{3}$.

Estudos que questionaram familiares de crianças com necessidades especiais de saúde, sobre quais profissionais os auxiliaram no processo de alta hospitalar, a equipe de enfermagem destacada. Entretanto, é importante destacar que a equipe de enfermagem é responsável pela efetivação de um plano assistencial voltado para as CRIANES e as suas famílias, uma vez que no retorno ao domicílio, a família se tornará o principal responsável pelos cuidados dessa criança. A equipe de enfermagem deve ser a grande aliada da família, considerando a complexidade do cuidado e os desafios que serão enfrentados no cotidiano ${ }^{4}$.

Considerando a especificidade dos cuidados necessários às crianças com necessidades especiais de saúde, percebe-se a necessidade que durante sua formação, o enfermeiro seja instrumentalizado para cuidar de crianças com deficiência e sua família. Quando falamos de criança com deficiência, é imprescindível o envolvimento de sua família, visto que é esta gerenciará e realizará o cuidado durante toda a vida da criança, podendo ser apoiada pela equipe de enfermagem ${ }^{5}$.

Diante do exposto, este estudo justifica-se pela necessidade de compreender como a formação acadêmica do profissional enfermeiro influencia na assistência prestada à criança com necessidades especiais e sua família. $O$ enfermeiro constitui-se peça fundamental para que a continuidade do cuidado à criança aconteça de forma eficaz, portanto, faz-se necessário que este profissional acumule competências para garantir uma assistência de qualidade às crianças com deficiência e suas famílias. Desta forma, este estudo tem o objetivo de analisar a percepção de enfermeiros frente ao cuidado de CRIANES e suas famílias em Unidades de Terapia Intensiva Neonatal e Pediátrica.

\section{MÉTODO}

Trata-se de um estudo descritivo-exploratório de abordagem qualitativa, uma vez que busca descobrir interações sociais que ainda são pouco conhecidas e pertencentes a grupos específicos ${ }^{6}$.

Pesquisa qualitativa, na qual buscou-se atender aos passos recomendados pelos Critérios Consolidados para Relatar uma Pesquisa Qualitativa (COREQ), o qual trata-se de um guia de pesquisa composto por 32 itens considerados necessários ao desenvolvimento de estudos qualitativos.

Os participantes deste estudo foram 10 profissionais enfermeiros, que atuam em Unidades de Terapia Intensiva Neonatal (UTIN) e Unidades de Terapia Intensiva Pediátrica (UTIP) de um hospital de referência em cuidados críticos à recém-nascidos e crianças situado na região Norte do Ceará.

Como critério de inclusão, foi utilizado: ser profissional graduado em enfermagem há no mínimo um ano, ou que possua experiência na assistência à criança com necessidades especiais de saúde em Unidades de Terapia 
Intensiva Neonatal ou Pediátrica. Os critérios de exclusão, foram profissionais que estavam de férias ou licença no período de coleta de dados.

Utilizou-se como instrumento de coleta de dados uma entrevista semiestruturada, que foram gravadas e, posteriormente transcritas para análise das informações. Dentre os questionamentos realizados aos participantes estão os seguintes: $O$ que é a criança com deficiência no ambiente de UTI? Como foi a sua primeira experiência de cuidado à criança com deficiência em uma UTI e sua família? Quais as dificuldades? Como vê o trabalho do enfermeiro no momento em que a família recebe o diagnóstico de uma deficiência na criança? Durante sua vivência como enfermeiro em UTI até o momento, como foi para você prestar assistência a uma criança com deficiência?

Para este estudo, optou-se pela análise de conteúdo temática, a qual objetiva à interpretação do material qualitativo afirmando uma descrição objetiva e sistemática trazendo toda a peculiaridade exposta no momento da coleta. A análise de conteúdo procura destacar todo o conteúdo manifesto na mensagem e o que ele representa ${ }^{7}$.

Após a transcrição das entrevistas iniciou-se a organização do material de base empírica. Foram realizadas leituras do material inicial a fim de conhecer o texto. As falas dos entrevistados foram codificadas por (EP) para os enfermeiros atuantes em UTI Pediátrica e (EN) para os enfermeiros atuantes em UTI Neonatal.

Por se tratar de um estudo que envolve seres humanos, esta pesquisa seguiu todas as normas da Resolução $n^{\circ} 466$ de 12 de dezembro de 2012 do Conselho
Nacional de Saúde, obtendo Parecer do CEP-UVA No. 3.3।3.774. Esta resolução baseia-se nos referenciais da bioética: autonomia, não maleficência, beneficência, justiça e equidade, dentre outros, além de afirmar todos os direitos e deveres dos participantes da pesquisa ${ }^{8}$.

\section{RESULTADOS E DISCUSSÕES}

As vivências de enfermeiros atuantes em unidades de terapia intensiva no cuidado à CRIANES e sua família, estão apresentadas neste estudo, primeiramente, pela caracterização do perfil dos enfermeiros participantes da pesquisa, tendo em vista a necessidade de conhecer aspectos formativos, que trazem importantes repercussões no cuidado à CRIANES e sua família no ambiente de UTI, e, posteriormente, pela análise dos depoimentos dos participantes que retratam suas experiências no cuidado à CRIANES e sua família em UTI pediátrica e neonatal.

A caracterização do perfil dos enfermeiros foi construída a partir das entrevistas, expondo as variáveis de idade, sexo, títulos de pós-graduação, tempo de atuação no serviço, regime de trabalho e outros vínculos empregatícios.

Os resultados da Tabela I mostram que apenas um enfermeiro possui idade de $4 \mathrm{I}$ a 50 anos, dois enfermeiros possuem idade de 20 a 30 anos, outros dois possuem de 51 a 60 anos e cinco possuem idade de 31 a 40 anos. Há uma prevalência do sexo feminino, sendo oito e apenas dois do sexo masculino.

Oito dos enfermeiros entrevistados possuem especialização e dois não possuem especialização e/ou residência. Quatro enfermeiros estão no serviço por um período de

TABELA 1 - Caracterização dos participantes da pesquisa. Sobral - CE. 2019.

\begin{tabular}{|c|c|c|}
\hline \multicolumn{2}{|l|}{ VARIÁVEL } & \multirow{2}{*}{$\begin{array}{l}\mathbf{N} \\
02 \\
05 \\
01 \\
02\end{array}$} \\
\hline Idade & $\begin{array}{l}20 \text { a } 30 \text { anos } \\
31 \text { a } 40 \text { anos } \\
41 \text { a } 50 \text { anos } \\
51 \text { a } 60 \text { anos }\end{array}$ & \\
\hline Sexo & $\begin{array}{l}\text { Masculino } \\
\text { Feminino }\end{array}$ & $\begin{array}{l}02 \\
08\end{array}$ \\
\hline Especialização e/ou Residência & $\begin{array}{l}\text { Especialização } \\
\text { Residência } \\
\text { Nenhum }\end{array}$ & $\begin{array}{l}08 \\
00 \\
02\end{array}$ \\
\hline Tempo de atuação no serviço & $\begin{array}{l}1 \text { ano } \\
2 \text { a } 3 \text { anos } \\
3 \text { a } 5 \text { anos } \\
>5 \text { anos }\end{array}$ & $\begin{array}{l}01 \\
01 \\
04 \\
04\end{array}$ \\
\hline Regime de trabalho & $\begin{array}{l}\text { CLT } \\
\text { Servidor Público } \\
\text { Cooperado }\end{array}$ & $\begin{array}{l}10 \\
00 \\
00\end{array}$ \\
\hline Vínculos empregatícios & $\begin{array}{l}\text { Apenas } 1 \text { vínculo } \\
2 \text { a } 3 \text { vínculos }\end{array}$ & $\begin{array}{l}03 \\
07\end{array}$ \\
\hline
\end{tabular}


3 a 5 anos, outros quatro trabalham no hospital há mais de 5 anos e apenas dois estão no serviço por um período menor que 3 anos. Todos os entrevistados trabalham em regime de CLT e faz-se importante destacar que sete enfermeiros possuem mais de um vínculo empregatício.

Corroborando com os resultados apresentados na Tabela I, estudos apontam os aspectos históricos e culturais que justificam a prevalência do sexo feminino na enfermagem, representados por uma afinidade histórica das mulheres com o cuidar, evidenciando-se que preconceitos de gênero restringiram a participação dos homens na profissão. Mas, embora a enfermagem seja construída culturalmente como prática predominantemente, feminina, os homens na profissão tornaram-se uma realidade cada vez mais comum, representando rupturas importantes com estereótipos de gênero relacionados à prática do cuidado?.

Outro importante resultado mostrado na Tabela I, envolve a predominância do número de enfermeiros que possuem mais de um vínculo empregatício, que neste estudo foi representado pelo total de sete enfermeiros. Os discursos dos enfermeiros revelaram a presença de sobrecarga relacionada a profissão, sendo a necessidade de obter mais de um vínculo empregatício, uma das principais causas de exaustão, baixo desempenho no trabalho e até mesmo problemas psicológicos.

Estudos apontam que dentre os principais fatores que tem gerado adoecimento profissional e a prestação de uma assistência insatisfatória ao paciente estão a dupla carga de trabalho, acompanhado de outros aspectos, como a desvalorização, a remuneração inadequada e a falta de investimento dos empregadores no local de trabalho ${ }^{10}$.

\section{PERCEPÇÕES DE ENFERMEIROS SOBRE O CUIDADO À CRIANES E SUA FAMÍLIA}

A percepção dos enfermeiros acerca do cuidado à criança e sua família está expressa em três categorias temáticas, a saber: (I) a criança com deficiência é aquela com sequelas; (2) o currículo de Enfermagem e a lacuna no cuidado à CRIANES e; (3) o trabalho do enfermeiro junto a CRIANES e sua família são essenciais.

\section{A CRIANÇA COM DEFICIÊNCIA É AQUELA COM SEQUELAS}

Esta categoria aponta as representações dos enfermeiros em relação a criança com deficiência e sua família em ambiente de UTI. Observou-se a predominância do modelo biomédico da deficiência nas falas dos entrevistados, onde a maioria percebe a criança com deficiência como um "paciente" que possui alguma morbidade ou sequela.

A criança com deficiência é toda aquela criança que possui alguma comorbidade, seja ela cerebral, motora, cardíaca. Enfim, esse é o meu en- tendimento sobre deficiência, é alguma comorbidade que a própria criança já possui, que ela não adquiriu em ambiente de UTI, mas que ela já veio com ele e com uma doença relacionada ou não a essa comorbidade. (EPI)

É aquela criança que tem doenças fora da sua idade habitual, essas patologias precisam de cuidados especiais mais rebuscado dentro da enfermagem, dentro do contexto da saúde e precisa de um aparado técnico e profissional adequado. (EP2)

A criança com deficiência é aquela criança que vai ter alguma sequela que vai interferir no seu desenvolvimento normal ou seja que futuramente ela vai ter não vai ter um desenvolvimento igual à das outras crianças da sua idade. (EP3)

A presença do modelo biomédico que define a saúde como ausência de doença e de defeitos em um corpo físico, faz-se presente nos dias de hoje, mesmo com o surgimento de novas definições ampliadas sobre o conceito de saúde. Este modelo considera a deficiência como consequência de uma doença ou outra circunstância que necessita de tratamento para que se consiga sua reabilitação ou cura. Tem como base as limitações funcionais que se encontram na pessoa e desconsidera o contexto social em que está inserida"l.

É importante destacar que os enfermeiros atuantes em UTIN definiram as crianças com deficiência relacionando às sequelas que estas possam adquirir, pois, neste setor, muitas vezes, os recém-nascidos ainda não possuem diagnósticos definitivos a respeito da deficiência.

Com o aperfeiçoamento da ciência, a sobrevivência das crianças em situações graves de saúde tem sido expandida, o que nos mostra a configuração de um novo público para o cuidado de enfermagem e a necessidade de qualificação da equipe de saúde para atender as demandas das CRIANES e de suas famílias.

Toda criança que necessita por muito tempo de ventilação mecânica, uma criança que a gente sabe que provavelmente vai ficar sequelada devido ao uso prolongado de aparelhos, uma criança que já desde o nascimento é diagnosticado com alguma síndrome, são essas crianças, asfixiados graves, são essas crianças que a gente sabe que vão ter que vão ter deficiência. (ENI)

A criança com deficiência que eu considero, são aqueles bebês que nascem com malformações congênitas, anencefalias, bebês anoxiados, a gente sabe que são bebêzinhos que vão ter sequelas, anomalias em geral, é isso que a gente considera aqui.(EN2) 
É uma criança especial... como eu trabalho aqui na NEO... aqui a deficiência que a gente fala... que eu posso dizer... são as sequelas que elas vão ter após a alta. (EN3)

A chegada de um filho é considerada um acontecimento importante para a família. No entanto, quando o recém-nascido sofre asfixia perinatal grave durante 0 nascimento, inicia-se um processo complexo de interação entre família, criança e profissionais de saúde, pois, os pais ao serem informados sobre a chegada de uma criança com síndrome, ou um possível atraso em seu desenvolvimento neuropsicomotor, começa-se um período desafiante de aceitação e interação da família com o recém-nascido ${ }^{12}$.

\section{O CURRÍCULO DE ENFERMAGEM E A LACUNA NO CUIDADO À CRIANES}

A formação de profissionais habilitados para cuidar de crianças com necessidades especiais de saúde e sua família é imprescindível para a inclusão deste público na sociedade. Sabemos que o enfermeiro atua durante todo o ciclo de vida da mulher e da criança, em todos os níveis de atenção à saúde. $O$ trabalho desenvolvido na Estratégia de Saúde da Família, acolhendo a mulher, especificamente, durante um período gestacional e por meio do pré-natal identificando-se possíveis fatores de risco para que a criança possa vir a ter algum tipo de deficiência e faz os encaminhamentos para promover o acesso a todos os serviços de saúde de acordo com as necessidades da mãe e da criança.

Quando questionados se tiveram alguma oportunidade durante a graduação de estudar sobre a assistência de enfermagem voltada às CRIANES e suas famílias, nove dos participantes relataram não ter contato com esta temática, fato que pode tornar a assistência à criança com deficiência incipiente.

... eu terminei minha graduação e vim direto para UTI pediátrica, eu não sabia muito como lidar, principalmente, por que os leitos aqui, a maioria são crianças que apresentam algum tipo de deficiência, então eu não sabia lidar nem com o paciente, nem com a família (EPI). ... não teve nenhuma preparação. $A$ faculdade é muito falha com relação a preparar a gente para o que iremos ver na assistência (EP2).

Na verdade, na faculdade a gente não via nada que fosse voltado para isso. A gente sabia que existia esse tipo de paciente com deficiência, mas não vi nada voltado para criança com deficiência. Eu acho que é algo que falta no currículo acadêmico (EP6).

Em alguns discursos percebeu-se que os enfermeiros relatavam sua não aproximação com a temática durante o estudo de conteúdos teóricos, contudo, em vivências práticas ocorreu a oportunidade prestar assistência a CRIANES e sua família.

Sobre assistência de enfermagem a criança com deficiência e sua família, eu lembro que foi muito marcante para a gente a visita a APAE, porque nos estágios da faculdade era mais aquela coisa de estágio observacional tinha pouca prática (EN I).

Em sala de aula, eu não recordo de ter visto nada sobre isso, embora no internato eu tenha encontrado algumas crianças com paralisia cerebral, algumas crianças com deficiência, mas na teoria mesmo, eu não me recordo de ter visto nada sobre isso(EN2)

...na época que eu fiz a faculdade a assistência de enfermagem não era muito direcionada para criança... sabe? se tinha uma certa dificuldade ...mas a gente via por alto e até mesmo na parte de estágios a gente tinha um estágio mas era em pediatria sabe? e a gente prestava essas informações as mães a respeito da doença, para todos os cuidados que era precisaria fazer em casa o preventivo para evitar que a criança voltasse para o hospital a gente tinha muito essa parte. Quanto ao cuidado em UTI, a gente não via muito... hoje está bem mais avançado (EP3).

Os participantes expressaram ainda a necessidade explorar conteúdos relacionados às crianças com deficiência, no sentido de qualificar a formação e, consequentemente, a assistência de enfermagem.

Eu acredito que na disciplina de saúde da criança deveria ser feito uma abordagem melhor dessas crianças, sobre os cuidados mais especificos, para que o estudante quando se deparar com essas crianças durante a sua vida profissional ele já estivesse mais preparado (EP 6).

Eu acho que deveriam inserir alguma disciplina que nos ajudasse... porque é uma realidade nossa (EP4).

... a gente tem visto muitos casos sobre crianças com deficiência, acho que deveria ter uma abordagem sobre isso, tanto na teoria como na prática, querendo ou não, é um paciente diferenciado, ele não vai ter os mesmos movimentos do outro, as mesmas funções, então acho que isso deveria ser estudado, acho que é uma forma de deixar as coisas mais naturais possíveis, acho que é a partir daí que as coisas vão começar a funcionar (EN 2)

O conhecimento incipiente e a invisibilidade da deficiência são notórias. Os discursos dos profissio- 
nais mostram que antes de trabalhar na assistência, não tinham contato com pessoas com deficiência. A lacuna nas formações acadêmicas sobre o assunto foi apontada como o motivo pelo qual isto acontece. A aproximação com a temática acontece por meio de programas de iniciação científica, participações em pesquisas, o que não é realidade de todos os alunos no meio acadêmico. Até mesmo em cursos de especialização na área, percebe-se uma abordagem distante da realidade ${ }^{13}$.

\section{O TRABALHO DO ENFERMEIRO JUNTO À CRIANES E SUA FAMÍLIA É ESSENCIAL}

Sabe-se que historicamente, todo o cuidado à criança é centralizado na mãe, o que causa uma sobrecarga para a mesma. Em todas as entrevistas, a mãe foi citada como a principal ou única cuidadora da criança.

...a gente tenta descentralizar o cuidado da mãe tentando fazer com que venha alguém da família...Então vem outra pessoa de escolha da própria mãe que seja alguém que ela diga: não eu acho que essa pessoa vai me ajudar nesses dias (EN I).

Corroborando com a temática expressa na fala de ENI, estudos evidenciam que mães de crianças com necessidades especiais de saúde são mais vulneráveis ao adoecimento mental do que as mães de crianças com desenvolvimento saudável ${ }^{14}$.

Neste estudo, evidencia-se a relevância do trabalho do enfermeiro junto à família da criança com necessidades especiais. $O$ enfermeiro atua nesta área fornecendo orientações quanto aos cuidados que a criança necessita, como também, informando a família quanto a existência da rede de apoio à criança.

$O$ trabalho do enfermeiro é a questão da orientação sobre os cuidados, se ele for traqueostomizado, tem a questão da aspiração, da alimentação por sonda enteral, dos sinais e sintomas de risco de broncoaspiração, de lesão por pressão, dependendo da deficiência né... claro... o acompanhamento pela estratégia de saúde da família, pela equipe multiprofissional, de todos os riscos que o bebê possa ter de acordo com a deficiência dele (EN2).

Um estudo apresentou que a rede de cuidados à CRIANES revelou-se complexa, constituída pela dimensão institucional e familiar, sendo a rede institucional composta por diversos profissionais da área da saúde e da educação. Relatando que os serviços de atenção primária foram considerados como de difícil acesso, apresentando dificuldades nos serviços de referência e contra referência, embora os serviços de pronto atendimento foram apontados como resolutivos. Evidenciou-se que a rede familiar está organizada, muitas vezes, em um cuidado solitário, predominantemente, feminino ${ }^{15}$.

Os discursos revelam que o enfermeiro é essencial para que a continuidade do cuidado ocorra efetivamente, uma vez que consegue identificar se a mãe está apta para realizar os cuidados domiciliares, e assim, junto com a equipe multiprofissional, planeja a alta hospitalar. Além de orientar a família quanto aos aspectos relacionados à reabilitação da criança diante do universo de necessidades e demandas de cuidado.

... aí a gente já vai preparando toda a família para o processo que eles vão ter que enfrentar não só de aprendizagem, como também, na justiça, porque o município muitas vezes não quer liberar os materiais que aquele paciente precisa. Ele vai precisar de uma cama hospitalar, de oxigênio e isso tudo é muito caro. $O$ paciente entrou para o domicílio, requer um custo e a prefeitura não quer pagar e aí elas sofrem não só para aprender os cuidados, mas também para levar aquela criança para casa, é todo um processo né, a gente vai preparando e aconselhando, tanto o enfermeiro, quanto o fisioterapeuta, todo processo de aspirar em casa, a gente vai explicando como é que aspira e sempre fazendo a observação se tá aspirando corretamente, ensina como é que faz administração dos medicamentos, administração de medicamentos por sonda, tudo isso elas tem que saber fazer antes de ir para casa... como é que faz a limpeza do traqueóstomo, como é que troca a fixação... a criança só recebe alta quando a gente já tá certificado de que elas estão fazendo isso corretamente, então o enfermeiro tem que observar essa parte aqui antes de ir para a alta (EP I).

Junto com a equipe multiprofissional, a gente tem uma responsabilidade muito grande, pois a gente tem que treinar a família e nós é quem vamos dizer ao médico se a mãe está apta ao não, por a gente está todos os dias com a criança e com a mãe a gente vai ver se ela está apta ao não. Ela tem que está preparada pra tudo... medicação, aspirar, ambuzar... (EP 4)

O enfermeiro pode atuar como educador em saúde, não somente repassando técnicas do cuidado em enfermagem para as mães de CRIANES, mas também, conscientizando-as sobre os direitos da criança. Pode-se perceber que algumas mães não conhecem os direitos que a criança possui, e as que os conhecem, definiram como um "favor"16.

O entrevistado EP 5 relatou em seu discurso que quando a orientação à família sobre as necessidades da criança é efetiva, gera impactos positivos como a dimi- 
nuição do tempo de internação hospitalar. Outro relato importante de EPI é que no momento que a mãe está perto da criança no leito, há uma melhora do estado de saúde da criança e da mãe.

Geralmente esses encefalopatas tem internação prolongada e todas a as mães são aptas a fazer o cuidado domiciliar e quem dá base pra elas fazerem isso é a equipe de enfermagem, elas chegam aqui muitas vezes sem saber fazer nada e saem sabendo fazer tudo, isso é maravilhoso. Isso só acontece na UTI, a gente já tem esse olhar para o futuro, sabemos que o paciente vai precisar desse cuidado em casa e então porque não vou habilitar a mãe a fazer esses cuidados? depois que elas já estão aptas, até a parte psicológica da mãe melhora, eles tem uma conexão muito grande. Quando mãe sai de perto eles hipossaturam, ficam hipoativos, a tendência é melhorar, pra que melhor poder cuidar do meu filho em casa e ainda diminui o tempo de internação hospitalar (EP 5).

Corroborando com o discurso de EP 5, em um estudo realizado com 20 pais e mães de recém-nascidos internados em UTIN, evidenciou que estes mostraram-se felizes ao interagir com seus filhos, dando significados aos sons e gestos emitidos pelo recém-nascido no momento da aproximação, visto que 19 participantes afirmaram que seus filhos percebiam a sua presença e reconheciam sua voz.Verificou-se que durante a primeira visita dos pais ao RN internado na UTIN, ocorre o reconhecimento por meio da voz, do toque e do olhar, colaborando para a desconstrução do medo em cuidar do filho. Nesta situação, percebeu-se que muitos pais necessitam de apoio para iniciar a relação afetiva com o $\mathrm{RN}$, portanto o profissional de saúde pode atuar na promoção deste cuidado, oferecendo apoio, esclarecendo dúvidas para que a relação en- tre os pais e os filhos se torne harmoniosa. Identificou-se que quando os pais são encorajados pelos profissionais a interagirem com o $\mathrm{RN}$ esta conexão entre ambos gera conforto e esperança, minimizando o sentimento de inutilidade, mediante o quadro de prematuridade ou doença ${ }^{17}$.

Há uma necessidade de recomendar alternativas de atenção à cuidadores de crianças hospitalizadas que necessitam de cuidados intensivos, bom como reconhecer aspectos psicológicos e orientar serviços que possam auxiliar nas dificuldades de interação com a criança. Neste aspecto, é fundamental a qualificação da equipe de saúde para fortalecer o binômio mãe e filho' ${ }^{18}$.

\section{CONSIDERAÇÕES FINAIS}

A realização desta pesquisa evidenciou a importância da inclusão da temática sobre CRIANES e sua família no currículo acadêmico da graduação em enfermagem, além da pluralidade do processo de cuidar do profissional enfermeiro na área de saúde da criança.

Ficou evidente a necessidade de expandir as vivências práticas durante a formação do enfermeiro, uma vez que este exerce papel relevante no desenvolvimento de competências do cuidador familiar para atender as demandas das crianças no processo de reabilitação.

Sugere-se que as instituições de ensino e os serviços de saúde promovam momentos de discussões que envolvam o universo vivenciado pelas CRIANES e suas famílias, com vistas a contribuir para a qualificação dos profissionais numa perspectiva humana e integral.

As pesquisas acerca desta temática têm avançado ao longo dos anos, contudo, observam-se desafios no que se refere à integração entre teoria e prática na formação. É preciso avançar em relação as práticas e políticas públicas direcionadas à CRIANES e sua família, para que a sobrecarga desta seja minimizada e possamos desenvolver um cuidado resolutivo, integrado e humanizado. 


\section{REFERÊNCIAS}

I. Brasil. Cartilha do censo de 2010: pessoas com deficiência. Brasília: Secretaria de Direitos Humanos da Presidência da República (SDH/PR) / Secretaria Nacional de Promoção dos Direitos da Pessoa com Deficiência (SNPD);2010.

2. McPherson MG, Arango P, Fox H, Lauver C, McManus M, Newacheck PW, et al. A new definition of children with special health care needs. Pediatrics. 1998; 102:137-41.

3. Luisada V, Fiamenghi Júnior GA, Carvalho SG, Madeira EAA, Assis SMB. Experiências de médicos ao comunicarem o diagnóstico da deficiência de bebês aos pais, 2015. [acesso em: 19 mar. 2020]; 8(3). Disponível em: http://revistaseletronicas.pucrs.br/ojs/index.php/faenfi/article/view/21769

4. Zamberlan KC, Neves ET, Cardoso CP, Silveira CV, Gomes MT. Cotidiano de cuidado da equipe de enfermagem às crianças com necessidades especiais de saúde hospitalizadas e suas famílias. Santa Maria. Dissertação de mestrado - Universidade Federal de Santa Maria; 2014.

5. Tavares T S. Implicações para o cuidado de enfermagem de egressos de unidade neonatal com condições crônicas. Revista Rene. 2016;17(5):659-667.

6. Minayo, MCS. O desafio do conhecimento: Pesquisa Qualitativa em Saúde. I2a. ed. São Paulo: Hucitec-Abrasco; 2010.

7. Bardin, L.Análise de conteúdo. Lisboa: Edições 70 Lda, 2009.

8. Conselho Nacional de Saúde. Resolução n 466 de 12 de dezembro de 2012. Diretrizes e normas reguladoras de pesquisas em seres humanas e a dignidade da pessoa humana. Diário Oficial da União 13 de jun 2013; seção I.

9. Coelho, E. Gênero, saúde e enfermagem. Rev. bras. Enferm. 2005;58(3):345-348.

10. Alvim et al. Relação entre processo de trabalho e adoecimento mental da equipe de enfermagem. Revista Fluminense de Extensão Universitária. 2017;07 (I):12-16.
1 I. Maior IML. Movimento político das pessoas com deficiência: Reflexões sobre a conquista de direito. Rev. Inc. Soc. 2017;10(2):28-36.

12. Milbrant VM, Siqueira HCH, Motta MGC, Amenstoy SC. Comunicação entre a equipe de saúde e a família da criança com asfixia perinatal grande. Rev Texto Contexto Enferm. 20II;20(4):726-734.

13. Fernandes ACL. Educando para a diversidade: A formação do enfermeiro com vistas à educação inclusiva. Mossoró. Dissertação de Mestrado - Universidade do Estado do Rio Grande do Norte; 2014.

14. Guerra CS, Dias MD, Ferreira-Filha MO, Andrade FB, Reichert APS, Araújo VS. Do sonho a realidade: Vivência de mães de filhos com deficiência. Rev Texto Contexto Enferm. 20I5;24(2):459-466.

15. Neves ET, Silveira A, Arrué AM, Pieszak GM, Zamberlan KC, Santos RP. Rede de cuidados de crianças com necessidades especiais de saúde. Rev. Texto e Contexto Enferm. 2015;24(2):399-406.

16. Milbrath VM, Siqueira HCH, Motta MGC, Amestoy SC, Resta DG, Soares DC, Freitag VL et. Direitos das crianças com necessidades especiais: situações de vulnerabilidade das famílias.J Nurs Health. 2016; I (I):27-37.

17. Frigo J, Zoche DAA, Palavro GL, Turatti LA, Neves ET, Schaefer TM. Percepções de pais de recém-nascidos prematuros em unidade de terapia intensiva neonatal. Rev Enferm UFSM. 20I5;5(I):58-68.

18. Nardi CGA, Rodrigues OMPR, Melchiori LE, Salgado MH, Tavano LDA. Bebês com sequência de Pierre Robin: saúde mental materna e relação mãe - bebê. Estudos de Psicologia I Campinas. 20I5;32(I) I29-I 40.

Recebido: 2020-06-25

Aceito: 2020-09-15 